\title{
Tissue acquisition and pancreatic masses: Which needle and which acquisition technique should be used?
}

\section{다)(우우}

\author{
Authors \\ Benedetto Mangiavillano ${ }^{1,2}$, Leonardo Sosa-Valencia ${ }^{3}$, Pierre Deprez $^{4}$, Pierre Eisendrath ${ }^{5}$, Carlos Robles-Medranda ${ }^{6}$, \\ Leonardo H. Eusebi ${ }^{7}$, Milena Di Leo ${ }^{8}$, Francesco Auriemma ${ }^{1}$, Mario Bianchetti ${ }^{1}$, Andrea Anderloni ${ }^{8}$, Silvia Carrara ${ }^{8}$, \\ Alessandro Repici ${ }^{2,8}$
}

Institutions

1 Gastrointestinal Endoscopy Unit - Humanitas Mater Domini - Castellanza (VA), Italy

2 Humamitas University

3 Ihu Strasbourg - Institute of Guided Image Surgery, Strasbourg, France

4 Department of Hepato-Gastroenterology, Cliniques universitaires Saint-Luc,

5 Endoscopy Unit Hopital Erasme, Brussels, Belgium

6 Instituto Ecuatoriano de Enfermedades Digestivas (IECED), Guayaquil, Ecuador

7 Endoscopy Unit, Department of Medical and Surgical Sciences, Sant'Orsola University Hospital, Bologna, Italy

8 Digestive Endoscopy Unit, Humanitas Clinical and Research Center, Rozzano (MI), Italy.

submitted 1.4 .2020

accepted after revision $\quad$ 17.6.2020

Bibliography

Endoscopy International Open 2020; 08: E1315-E1320

DOI 10.1055/a-1221-4578

ISSN 2364-3722

(c) 2020. The Author(s).

This is an open access article published by Thieme under the terms of the Creative Commons Attribution-NonDerivative-NonCommercial License, permitting copying and reproduction so long as the original work is given appropriate credit. Contents may not be used for commecial purposes, or adapted, remixed, transformed or built upon. (https://creativecommons.org/licenses/by-nc-nd/4.0/)
Corresponding author

Benedetto Mangiavillano, MD, Gastrointestinal Endoscopy

Unit, Humanitas - Mater Domini, Via Gerenzano n.2, 21053-

Castellanza (VA)

Fax: +00390331476210

bennymangiavillano@gmail.com

\section{ABSTRACT}

Background and study aims Pancreatic cancer represents the fourth most common cause of cancer-related deaths in Western countries and the need of a low-risk investigation to obtain an accurate histopathological diagnosis has become increasingly pressing. Endoscopic ultrasonography (EUS) with fine-needle aspiration (FNA) is the standard method for obtaining samples from pancreatic masses. In recent years, there has been an increasing need to obtain histological specimens during EUS procedures, rather than cytological ones, to guide oncological treatment options, leading to the so-call "FNB concept." Different needles have been developed for fine-needle biopsy (FNB) in recent years, enabling acquisition of larger specimens on which to perform histological and molecular analyses. The aim of this narrative review was to assess the role of EUS-guided FNA and FNB in patients with pancreatic masses, and to identify which needle and which acquisition technique should be used to improve tissue acquisition.

\section{Evolution from FNA to FNB}

Pancreatic cancer represents the fourth most common cause of cancer-related deaths in Western countries, with a 5-year survival rate of $6 \%[1-3]$.To improve treatment for these patients and to increase their survival, the need for a low-risk investigation has become essential to obtain an accurate histopathological diagnosis [4]. The standard method for sampling pancreatic masses is fine-needle aspiration (FNA) performed under endoscopic ultrasonography (EUS) guidance. The sensitivity, specificity and diagnostic accuracy of FNA for malignant cytological diagnosis have been reported to range between $85 \%$ to $95 \%$,
$95 \%$ to $98 \%$ and $78 \%$ to $95 \%$, respectively $[5,6]$. Several studies published in recent years aimed to identify factors related to non-diagnostic or false-negative EUS-FNA sampling; moreover, to improve its diagnostic yield, different sizes of needles and tissue acquisition (TA) techniques, such as fanning technique, slow-pull stylet extraction or suction technique, have been implemented.

Despite data reporting a high sensitivity and specificity, more accurate diagnostic results with EUS-FNA have been linked to the availability of rapid on-site evaluation (ROSE). However, studies evaluating the diagnostic efficacy of EUS-FNA plus 
ROSE showed a sensitivity and specificity of $83 \%(95 \% \mathrm{Cl}, 64 \%$ to $93 \%$ ) and $98 \%(95 \% \mathrm{Cl}, 80 \%$ to $100 \%)$, compared with $65 \%$ $(95 \% \mathrm{Cl}, 57 \%$ to $73 \%)$ and $94 \%(95 \% \mathrm{Cl}, 31 \%$ to $100 \%)$ when ROSE was not available [7,8]. Thus, no significant difference regarding diagnostic accuracy was found between the two groups, with no clear benefit from the use of ROSE. Recently, a systematic review with meta-analysis evaluated the pooled diagnostic accuracy of a repeat EUS-FNA (rEUS-FNA) following a previous non-diagnostic result, demonstrating that rEUS-FNA had an optimal pooled specificity ( $97 \%$ ) with high pooled sensitivity $(78 \%)$ for diagnosis of pancreatic malignancies. In particular, the pooled sensitivity increased from $65 \%(95 \% \mathrm{Cl} 57-$ $73)$ without ROSE to $83 \%(95 \% \mathrm{Cl} 64-93)$ when ROSE was available, confirming the beneficial role of ROSE in this setting to increase the amount of definitive diagnoses [9].

Another limit of EUS-FNA is that it does not allow to obtain a specimen adequate for histological evaluation; therefore, for some pancreatic masses, such as in the presence of autoimmune pancreatitis, a cytological examination obtained with FNA is of limited value [10].

During recent years, there has been an increasing need to obtain histological specimens during EUS procedures, rather than cytological samples, to guide oncological treatment options. Therefore, the need for TA needles with different shapes and new configurations has led to the concept of fine-needle biopsy (FNB). Several needles for FNB are available with different design and technical features, such as needles with reverse bevel, fork tip or Franseen tip. A histological specimen could avoid the need of a cytopathologist in the endoscopic room, reducing procedures time, number of passes and additional costs of a possible repeated EUS-FNA, in case of an inconclusive diagnosis.

\section{Is FNB better than FNA?}

Different articles comparing FNA vs FNB have been published in recent years. Recently, in a prospective comparison study, conducted in a consecutive cohort of 36 patients affected by pancreatic cancer who underwent EUS-TA with $22 \mathrm{G}$ FNA and $22 \mathrm{G}$ FNB, Tian et al. showed that the number of passes needed to obtain a diagnosis was significantly lower for patients who underwent EUS-FNB (1.11 vs 1.83); in particular, the proportion of diagnoses of malignancies obtained with just one needle pass were significantly higher in the FNB group ( $80 \%$ vs $66.67 \%$ ) [11].

Recently, promising results have been reported by a randomized controlled trial, analyzing tissue and molecular diagnostic yield of FNA and FNB. Comparing the two techniques, median total tissue area for each specimen was significantly higher for FNB (1.9 vs $5.2 \mathrm{~mm}^{2}$ ), with a consequent higher onsite diagnostic rate. One of the most important novelties arising from this study was that quantification of nucleic acid obtained from the samples was higher in patients who underwent FNB compared to FNA, regarding both DNA and RNA extraction. Indeed, EUS-FNB can obtain more tissue with higher content of nucleic acid allowing downstream genomics applications such as nucleotide genomic sequence assay (NGS). Because it is a convenient and safe method for obtaining tumor samples for genetic analysis, EUS-FNB could play a pivotal role in guiding new genomics therapies [12].

Other comparative studies showed similar results, in terms of fewer needle passes required to achieve adequate samples and more core TA with a single pass in favor of FNB. A recent retrospective study from Varadarajulu et al., which included more than 3000 patients, showed that significantly fewer passes were needed to obtain a correct diagnosis using FNB compared to FNA ( 1 vs 2; $P<0.001$ ), with an increased diagnostic yield on cell-block using FNB (92.3\% vs $71.1 \%$; $P<0.001)$ and an overall superior performance for pancreatic lesions $(P<$ $0.001)$ [13].

On the basis of the available literature, FNB has shown a diagnostic yield of more than $90 \%$ [14]. Moreover, the diagnostic performance of FNB sampling of pancreatic masses was significantly better than FNA sampling, and was also associated with ease of diagnosis and shorter viewing times by the pathologists [15]. As a result, presence of an onsite cytopathologist is not required during EUS-FNB sampling, reducing the overall duration of the procedures.

Similar results in terms of diagnostic yield and specimen adequacy for a correct diagnosis were also reported with use of $25 \mathrm{G}$ needles compared to larger ones; however, despite the high diagnostic yield of $25 \mathrm{G}$ needles, the smaller samples obtained with these needles could represent a limit considering advances in new oncological and molecular discoveries related to pancreatic cancer [16].

In the near future, FNA needles will probably be used only for selected cases, such as patients with complex anatomy or with a high risk of bleeding (portal hypertension, coagulopathies, and deep arterial or venous thrombosis). FNA needles could also be used for therapeutic EUS procedures, such as contrast injection into the pancreatic or bile ducts, release of a guidewire, or injection therapies [17].

\section{Which size needle should be used to sample pancreatic masses?}

The two most important variables influencing the diagnostic accuracy of EUS-TA of pancreatic masses are the type of needle (FNA and FNB), as previously discussed, and the size (19G, 20G, $22 \mathrm{G}$ or $25 \mathrm{G}$ ). Several types and designs of FNB needles are currently available [18]. The first FNB needle was launched in 2011 (ProCore - Wilson-Cook Medical Inc., United States); its main characteristic was the distal reversed bevel design and different sizes of needles were available for sampling: 19 G, 22 G and 25 G. A second-generation $20 \mathrm{G}$ needle with a proximal forward bevel design and "Menghini" tip type was launched in 2015: the EchoTip ProCore HD Ultrasound Biopsy Needle (Wilson-Cook Medical Inc., United States).

Recently, two different types of FNB needles have been introduced in clinical endoscopic practice: one with fork-tip design (SharkCore, Medtronic, Minneapolis, Minnesota, United States), and another with Franseen tip design (Acquire, Boston Scientific, Natick, Massachusetts, United States). A large network meta-analysis from Facciorusso et al. compared different sizes and types of needles for EUS tissue-acquisition, evaluating 
a total of 2711 patients. The meta-analysis showed that no EUSguided tissue sampling technique was superior to another, regardless of needle type (FNA vs FNB) or size (19G vs $22 \mathrm{G}$ vs 25 G). Moreover, no difference was found between 25G FNA and 22G FNA needles (relative risk [RR], 1.03; $95 \%$ confidence interval [Cl], $0.91-1.17$ ) and between $22 \mathrm{G} \mathrm{FNB}$ and $22 \mathrm{G}$ FNA needles (RR, 1.03; $95 \% \mathrm{Cl}, 0.89-1.18$ ) in terms of diagnostic accuracy, sample adequacy and histologic core procurement [19].

Diagnostic performance of the 20G Procore FNB needle was evaluated in a prospective, randomized, multicenter clinical trial compared to the $25 \mathrm{G}$ FNB needles for sampling of solid pancreatic lesions. This study included 88 patients and showed a significantly higher procurement rate of core biopsy specimens among the 20G FNB needle group (41/45, 91.1\%) compared to the 25G FNB needle group $(32 / 43,74.4 \%, P=0.037)$. No significant differences were observed in the overall diagnostic accuracy between the $20 \mathrm{G}$ FNB needle $(40 / 45,88.9 \%)$ and the $25 \mathrm{G}$ FNB $(34 / 43,79.1 \%, P=0.208)$. Therefore, although both FNB needles provided high overall diagnostic accuracy, the reliability of the $20 \mathrm{G}$ FNB needle was superior to the $25 \mathrm{G}$ FNB when retrieving samples for histological analysis [19], and should be preferred when sampling pancreatic masses.

A recent observational study comparing the $20 \mathrm{G}$ Procore vs the $22 \mathrm{G}$ Acquire needles for EUS-FNB of solid pancreatic masses, conducted on 68 patients, showed that histological diagnosis on core biopsy specimens was obtained in 28 of 34 patients ( $82 \%$ ) in the $20 \mathrm{G}$ group and in 33 of 34 patients (97\%) in the $22 \mathrm{G}$ group $(P=0.1)$. Mean cumulative length of tissue core biopsies per needle pass was significantly higher with the $22 \mathrm{G}$ needle with $8.2 \pm 4.2 \mathrm{~mm}$ versus $4.2 \pm 3.8 \mathrm{~mm}$ for the $20 \mathrm{G}$ needle $(P<.01)$, in the absence of intraobserver and inter-observer variability [21]. In our opinion, thinner needles (22 G vs. $20 \mathrm{G}$ ) may be easier to maneuver, especially during EUS-FNB of pancreatic masses in the head or the uncinate process, and the EUS scope is bent into the duodenum. Several new TA needles currently exist and none of them has shown a significant superiority in prospective controlled trials. The aim of EUS sampling should be to obtain an adequate sample for diagnosis in more than $90 \%$ of cases, and allow to performance of immunohistochemistry and evaluation of tumor markers, if necessary, to ensure the most appropriate oncological treatment for patients ( Fig.1).

\section{Is on-site evaluation necessary during FNB procedures?}

As previously reported, in some settings, such as during repeat EUS-FNA, ROSE can increase diagnostic accuracy. To evaluate its role during FNB sampling, a multicenter, randomized, noninferiority trial was conducted in 16 international centers, including a large prospective cohort of patients. The authors randomized 800 patients with solid pancreatic masses to sampling with EUS-FNB plus ROSE or EUS-FNB alone, demonstrating the non-inferiority of FNB alone [22].

With the advent of FNB, macroscopic on-site evaluation (MOSE) of the specimen by the endosonographer has been proposed as an alternative to $\operatorname{ROSE}[23,24]$. A recent retrospective

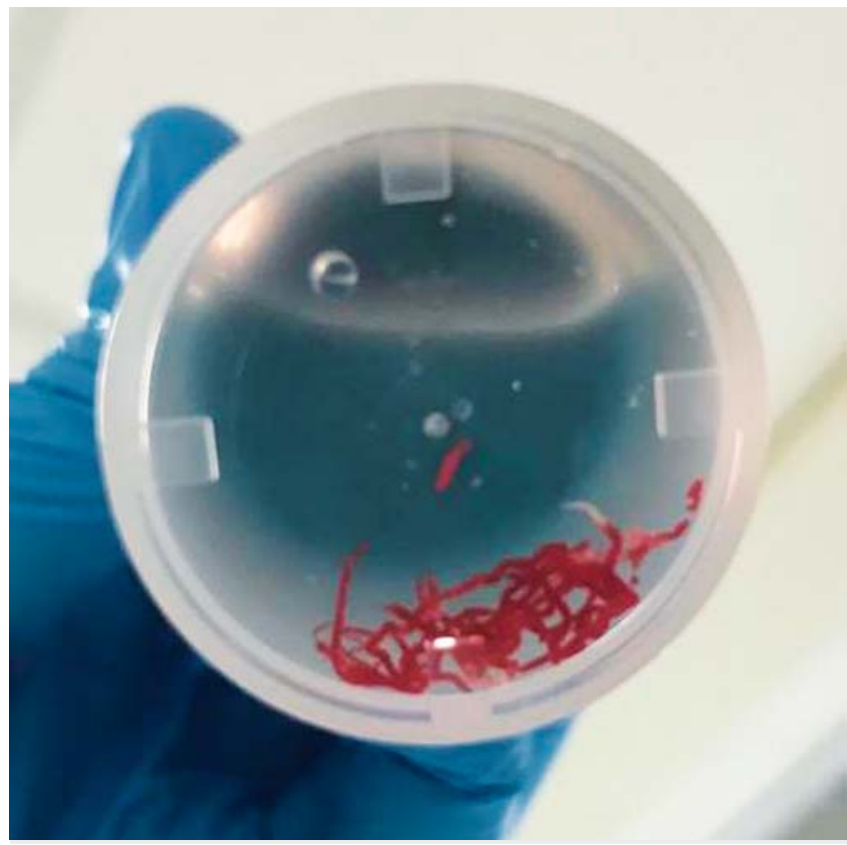

- Fig. 1 Specimen of a solid pancreatic mass obtained after two passes with a 22G FNB needle.

study including a cohort of 54 patients who underwent TA with a 22G Franseen-tip needle, showed an overall diagnostic accuracy of $94 \%$ adding MOSE to the FNB sampling. Moreover, the reported sensitivity, specificity, positive predictive value, and negative predictive value for malignancy were $92 \%, 100 \%$, $100 \%$, and $81 \%$, respectively, with no adverse events [25].

\section{Which acquisition technique should be used for sampling pancreatic masses?}

\section{Techniques for specimen acquisition}

There is no a standard technique for TA both for FNA and FNB. Different features influence the acquisition technique, such as the size of the pancreatic lesion, its location, the size and type of needle, and the availability of the ROSE [26-28].

Three main techniques have been described, and endosonographers usually choose which technique to use based on their personal experience. The first technique to have been described was the standard suction technique (SST). Briefly, after the needle tip is placed inside the target lesion, the stylet is pulled-back completely and a syringe, with high negative pressure, is mounted on the handle of the needle. The suction induced by the negative pressure facilitates entry of tissue into the needle. This technique is generally used for FNA but may damage the cellular structure of the tissue and cause considerable blood contamination of the specimen [29].

The second technique is the stylet slow-pull (SSP) technique. While the endosonographer performs to-and-fro movements with the needle inside the lesion, the stylet is slowly removed to create negative pressure, which allows the tissue to enter the needle $[30,31]$. The low negative pressure induced by slow- 
ly removing the stylet avoids damaging the specimen [32] and reduces blood contamination of the sample [33-35].

The third is the non-suction technique (NST) after stylet removal. After puncturing the targeted lesions, the stylet is completely removed and the needle is moved to-and-fro within the lesion, without a negative pressure syringe mounted onto the needle handle [36].

Recently, the three TA techniques were compared in a cohort of 50 patients, without using ROSE. The study showed that the rate of a good or excellent proportion of cellularity in the sample was highest when the SSP technique was used compared to the standard suction and non-suction techniques (SSP $72 \%$ vs SST $60 \%$ vs NST $50 \%, P=.049$ ). A > $25 \%$ rate of blood contamination was more prevalent in patients in which TA was performed with the standard suction technique (SSP $30 \%$ vs SST $42 \%$ vs NST $10 \%, P=.009$ ). The rate of adequate core-TA was not significantly different among the three groups (SSP 52 $\%$ vs SST $34 \%$ vs NST $50 \%, P=.140$ ).

Use of the SSP technique and tumor size $>40 \mathrm{~mm}$ were favorable factors for diagnostic adequacy [37].

Another TA technique has also recently been proposed, the wet-suction (WS) technique. This technique, rarely used, consists on irrigating the needle with saline solution to replace the air. The saline solution, being liquid, is less compressible than air, allowing better transmission of the negative pressure to the tip of the needle [38]. A RCT compared the WS to the standard suction technique during EUS-FNA in patients with solid masses and showed that WS improved both sample adequacy and quality [39].

\section{Techniques for targeting lesions}

Different techniques have been proposed for targeting lesions during TA. The standard technique (ST) consists of placing the tip of the needle within the lesion, moving the needle to-andfro on the same axis, regardless of the TA technique applied (standard suction, slow-pull, wet or non-suction technique).

Two other techniques, the fanning technique (FT) and the torque technique (TT), have been proposed to establish accurate diagnosis with fewer needle passes, resulting in shorter procedure duration and lower sedation requirement to carry out the procedure. FT consists of placing the needle in four different areas of the lesion by using the "up-down" wheel of the scope, applying to-and-fro movements four times in each area to procure tissue $(4 \times 4)$. This technique was initially proposed by Bang et al. who conducted a RCT comparing the two techniques. No significant differences were found regarding diagnostic accuracy $(76.9 \%$ vs. $96.4 \%$; $P=0.05)$, technical failure, or complication rates; however, there was a significant difference between the ST and FT in both the number of passes needed to establish a diagnosis and the percentage of patients in whom a diagnosis was achieved with the first needle pass (57.7\% vs. $85.7 \% ; P=0.02$ ) [40].

During the TT, the needle is first advanced into the lateral margin of the targeted lesion. Then, the operator performs repetitive to-and-fro movements while twisting the endoscope without using the left/right wheel. This technique was recently compared to the ST, showing significant differences between the groups regarding the procurement rate for the histologic cores and optimal quality cores (ST vs TT: $87.1 \%$ vs $98.4 \%, P=$ 0.038 and $79.0 \%$ vs $93.5 \%, P=0.037$ ). Sensitivity, specificity, positive predictive value, and negative predictive values for EUS-FNB were $85.5 \%, 100 \%, 100 \%$, and $46.7 \%$, respectively, using the ST, and $96.5 \%, 100 \%, 100 \%$, and $71.4 \%$ for the TT. Diagnostic accuracy of the ST and TT was $87.1 \%$ and $96.8 \%$, respectively. In conclusion, the TT for EUS-TA offered acceptable technical feasibility and superior diagnostic performance, including optimal histologic core procurement, compared with the ST [41]. No study comparing the FT vs TT has yet been published. Nevertheless, most endosonographers combine these three techniques based on their personal experience and on the characteristic of the lesion being targeted.

\section{Conclusions}

On the basis of the published data, FNB has proven to be superior to FNA for EUS-TA of pancreatic masses, as it makes it possible to obtain histological specimens that do not require ROSE, reducing the number of needle passes and procedure duration. FNB 22G needles seem to be the best option for sampling pancreatic masses, however, 25G needles can be used for challenging targets, such as small or hypervascular lesions. The fanning technique should be used to obtain specimens from different areas of the target lesion, and the stylet slow-pull technique also should be used to apply a low negative pressure, avoiding fragmentation of the specimen. Finally, FNB-acquired specimens should be evaluated by MOSE to limit needle passes ( Fig. 2).

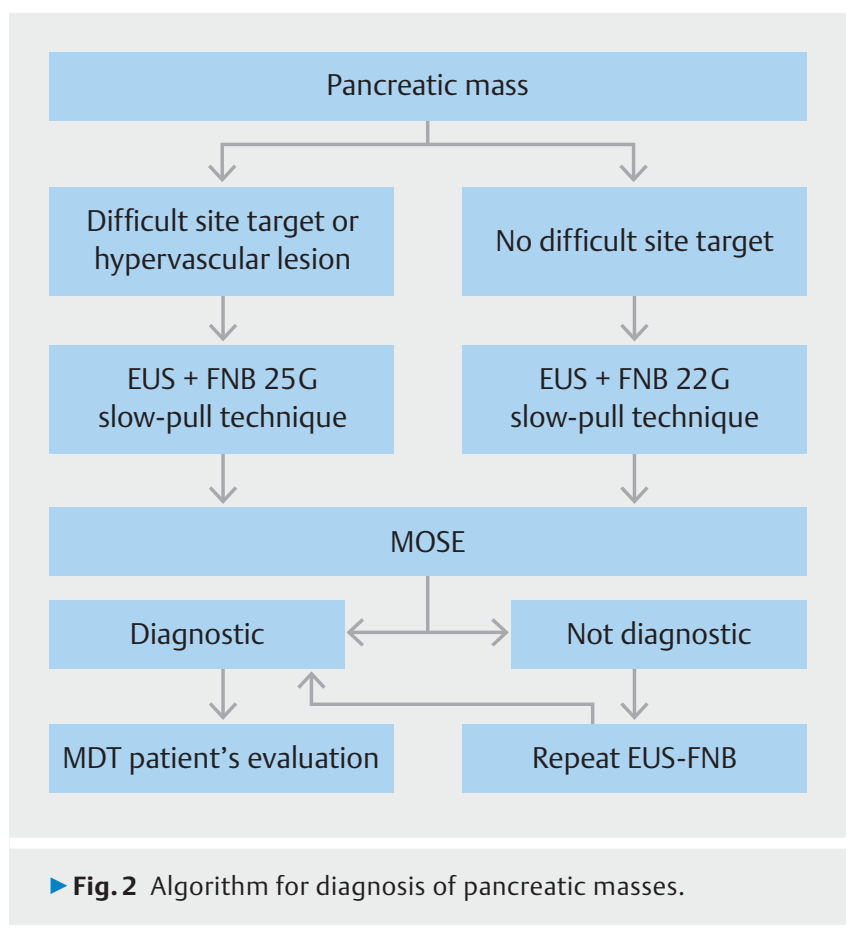




\section{Acknowledgments}

Dr. Mangiavillano thank Dr. Shyam Varadarajulu, who changed the panorama of endoscopic ultrasonography with his innovations and with his research in this field; Prof. Laurent Palazzo, who taught him diagnostic and therapeutic EUS; and Prof. Todd $\mathrm{H}$. Baron, who spent time with him me in Paris at the time when heneeded to learn EUS.

\section{Competing interests}

The authors declare that they have no conflict of interest.

\section{References}

[1] Maitra A, Hruban RH. Pancreatic cancer. Annu Rev Pathol 2008; 3: 157-188

[2] Cui X, Zhang Y, Yang J et al. ZIP4 confers resistance to zinc deficiencyinduced apoptosis in pancreatic cancer. Cell Cycle 2014; 13: 11801186

[3] Pietryga JA, Morgan DE. Imaging preoperatively for pancreatic adenocarcinoma. J Gastrointest Oncol 2015; 6: 343-357

[4] Del Chiaro M, Segersvard R, Lohr M et al. Early detection and prevention of pancreatic cancer: is it really possible today? World J Gastroenterol 2014; 20: 12118-12131

[5] Ngamruengphong S, Li F, Zhou Y et al. EUS and survival in patients with pancreatic cancer: a population-based study. Gastrointest Endosc 2010; 72: 78-83

[6] Othman MO, Wallace MB. The role of endoscopic ultrasonography in the diagnosis and management of pancreatic cancer. Gastroenterol Clin North Am 2012; 41: 179-188

[7] Kappelle WFW, Van Leerdam ME, Schwartz MP et al. Rapid on-site evaluation during endoscopic ultrasound-guided fine-needle aspiration of lymph nodes does not increase diagnostic yield: A randomized, multicenter trial. Am J Gastroenterol 2018; 113: 677-685

[8] Fabbri C, Fuccio L, Fornelli A et al. The presence of rapid on-site evaluation did not increase the adequacy and diagnostic accuracy of endoscopic ultrasound-guided tissue acquisition of solid pancreatic lesions with core needle. Surg Endosc 2017; 31: 225-230

[9] Lisotti A, Frazzoni L, Fuccio L et al. Repeated EUS-FNA of pancreatic masses after nondiagnostic or inconclusive results: systematic review and meta-analysis. Gastrointest Endosc 2020; 91: 1234-1241.e4

[10] Mizuno N, Bhatia V, Hosoda W et al. Histological diagnosis of autoimmune pancreatitis using EUS-guided trucut biopsy: a comparison study with EUS-FNA. J Gastroenterol 2009; 44: 742-750

[11] Tian L, Tang AL, Zhang L et al. Evaluation of 22G fine-needle aspiration (FNA) versus fine-needle biopsy (FNB) for endoscopic ultrasoundguided sampling of pancreatic lesions: a prospective comparison study. Surg Endosc 2018; 32: 3533-3539

[12] Asokkumar R, Yung Ka C, Loh T et al. Comparison of tissue and molecular yield between fine-needle biopsy (FNB) and fine-needle aspiration (FNA): a randomized study. Endosc Int Open 2019; 7: E955-E963

[13] Bang JY, Kirtane S, Krall K et al. In memoriam: Fine-needle aspiration, birth: Fine-needle biopsy: The changing trend in endoscopic ultrasound-guided tissue acquisition. Dig Endosc 2019; 31: 197-202

[14] Ishigaki K, Nakai Y, Oyama H et al. Endoscopic ultrasound-guided tissue acquisition by 22-Gauge Franseen and standard needles for solid pancreatic lesions. Gut Liver 2020: doi:10.5009/gnl19171 [Epub ahead of print]
[15] Oppong KW, Bekkali NLH, Leeds JS et al. Fork-tip needle biopsy versus fine-needle aspiration in endoscopic ultrasound-guided sampling of solid pancreatic masses: a randomized crossover study. Endoscopy 2020; 52: 454-461

[16] Conti CB, Cereatti F, Grassia R. Endoscopic ultrasound-guided sampling of solid pancreatic masses: the fine needle aspiration or fine needle biopsy dilemma. Is the best needle yet to come? World J Gastrointest Endosc 2019; 11: 454-471

[17] Robles-Medranda C, Oleas R, Valero M et al. Endoscopic ultrasonography-guided deployment of embolization coils and cyanoacrylate injection in gastric varices versus coiling alone: a randomized trial. Endoscopy 2020; 52: 268-275

[18] James TW, Baron TH. A comprehensive review of endoscopic ultrasound core biopsy needles. Expert Rev Med Devices 2018; 15: 127135

[19] Facciorusso A, Wani S, Triantafyllou K et al. Comparative accuracy of needle sizes and designs for EUS tissue sampling of solid pancreatic masses: a network meta-analysis. Gastrointest Endosc 2019; 90: 893903

[20] Cho E, Park CH, Kim TH et al. A prospective, randomized, multicenter clinical trial comparing 25-gauge and 20-gauge biopsy needles for endoscopic ultrasound-guided sampling of solid pancreatic lesions. Surg Endosc 2020; 34: 1310-1317

[21] Karsenti D, Tharsis G, Zeitoun JD et al. Comparison of 20-gauge Procore ${ }^{\circledR}$ and 22-gauge Acquire ${ }^{\circledR}$ needles for EUS-FNB of solid pancreatic masses: an observational study. Scand J Gastroenterol 2019; 54: 499-505

[22] Crinò SF, Manfrin E, Scarpa A et al. EUS-FNB with or without on-site evaluation for the diagnosis of solid pancreatic lesions (FROSENOR): Protocol for a multicenter randomized non-inferiority trial. Dig Liver Dis 2019; 51: 901-906

[23] Iwashita T, Yasuda I, Mukai T et al. Macroscopic on-site quality evaluation of biopsy specimens to improve the diagnostic accuracy during EUS-guided FNA using a 19-gauge needle for solid lesions: a singlecenter prospective pilot study (MOSE study). Gastrointest Endosc 2015; 81: 177-185

[24] Han P, Che D, Pallav K et al. Models of the cutting edge geometry of medical needles with applications to needle design. Int J Mech Sci 2012; 65: 157-67

[25] Leung Ki EL, Lemaistre Al, Fumex F et al. Macroscopic onsite evaluation using endoscopic ultrasound fine needle biopsy as an alternative to rapid onsite evaluation. Endosc Int Open 2019; 7: E189-E194

[26] Varadarajulu S, Fockens P, Hawes RH. Best practices in endoscopic ultrasound-guided fine-needle aspiration. Clin Gastroenterol Hepatol 2012; 10: 697-703

[27] Bang JY, Hebert-Magee S, Trevino J et al. Randomized trial comparing the 22-gauge aspiration and 22-gauge biopsy needles for EUS-guided sampling of solid pancreatic masses. Gastrointest Endosc 2012; 76: 321-327

[28] Tadic M, Stoos-Veic T, Kusec R. Endoscopic ultrasound guided fine needle aspiration and useful ancillary methods. World J Gastroenterol 2014; 20: 14292-14300

[29] Wani S. Basic techniques in endoscopic ultrasound-guided fine-needle aspiration: role of a stylet and suction. Endosc Ultrasound 2014; 3 : $17-21$

[30] Kudo T, Kawakami H, Hayashi T et al. High and low negative pressure suction techniques in EUS-guided fine-needle tissue acquisition by using 25-gauge needles: a multicenter, prospective, randomized, controlled trial. Gastrointest Endosc 2014; 80: 1030-1037

[31] Nakai $\mathrm{Y}$, Isayama $\mathrm{H}$, Chang $\mathrm{K}$ ] et al. Slow pull versus suction in endoscopic ultrasound-guided fine-needle aspiration of pancreatic solid masses. Dig Dis Sci 2014; 59: 1578-1585 
[32] Matsubayashi H, Matsui T, Yabuuchi Y et al. Endoscopic ultrasonography guided-fine needle aspiration for the diagnosis of solid pancreaticobiliary lesions: Clinical aspects to improve the diagnosis. World J Gastroenterol 2016; 22: 628-640

[33] Chen JY, Ding QY, Lv Y et al. Slow-pull and different conventional suction techniques in endoscopic ultrasound- guided fine-needle aspiration of pancreatic solid lesions using 22-gauge needles. World J Gastroenterol 2016; 22: 8790-8797

[34] Wang J, Wu X, Yin P et al. Comparing endoscopic ultrasound (EUS)guided fine needle aspiration (FNA) versus fine needle biopsy (FNB) in the diagnosis of solid lesions: study protocol for a randomized controlled trial. Trials 2016; 17: 198

[35] Kin T, Katanuma A, Yane K et al. Diagnostic ability of EUS-FNA for pancreatic solid lesions with conventional 22-gauge needle using the slow pull technique: a prospective study. Scand J Gastroenterol 2015; 50: $900-907$

[36] Sahai AV, Paquin SC, Gariepy G. A prospective comparison of endoscopic ultrasound-guided fine needle aspiration results obtained in the same lesion, with and without the needle stylet. Endoscopy 2010; 42: 900-903
[37] Lee KY, Cho HD, Hwangbo Y et al. Efficacy of 3 fine-needle biopsy techniques for suspected pancreatic malignancies in the absence of an on-site cytopathologist. Gastrointest Endosc 2019; 89: 825-831

[38] Villa NA, Berzosa M, Wallace MB et al. Endoscopic ultrasound-guided fine needle aspiration: The wet suction technique. Endosc Ultrasound 2016; 5: 17-20

[39] Attam R, Arain MA, Bloechl S] et al. "Wet suction technique (WEST)": a novel way to enhance the quality of EUS-FNA aspirate. Results of a prospective, single-blind, randomized, controlled trial using a 22gauge needle for EUS-FNA of solid lesions. Gastrointest Endosc 2015; 81: 1401-1407

[40] Bang JY, Magee SH, Ramesh J et al. Randomized trial comparing fanning with standard technique for endoscopic ultrasound-guided fineneedle aspiration of solid pancreatic mass lesions. Endoscopy 2013; 45: $445-450$

[41] Park SW, Lee SS, Song T] et al. The diagnostic performance of novel torque technique for endoscopic ultrasound-guided tissue acquisition in solid pancreatic lesions: A prospective randomized controlled trial. Gastroenterol Hepatol 2020; 35: 508-515 